regulate the $T$ and $B$ cell responses that effective vaccines seek to generate.

Address correspondence to: Sallie R. Permar, Human Vaccine Institute, Duke University Medical Center, Box 103020, Durham, North Carolina 27710, USA. Phone: 919.684.2551; Fax: 919.668.4859; E-mail: sallie.permar@duke.edu.

1. Excler JL, Robb ML, Kim JH. HIV-1 vaccines: challenges and new perspectives. Hum Vaccin Immunother. doi: $10.4161 /$ hv.28462.

2. Haynes BF, McElrath MJ. Progress in HIV-1 vaccine development. Curr Opin HIV AIDS. 2013; 8(4):326-332.

3. Moody MA, et al. Toll-like receptor 7/8 (TLR7/8) and TLR9 agonists cooperate to enhance HIV-1 envelope antibody responses in rhesus macaques. JVirol. 2014;88(6):3329-3339.

4. Buffa V, Klein K, Fischetti L, Shattock RJ. Evaluation of TLR agonists as potential mucosal adjuvants for HIV gp140 and tetanus toxoid in mice. PLoS One. 2012;7(12):e50529.

5. Fouda GG, et al. Mucosal immunization of lactating female rhesus monkeys with a transmitted/ founder HIV-1 envelope induces strong Envspecific IgA antibody responses in breast milk. J Virol. 2013;87(12):6986-6999.

6. Staats HF, et al. Cytokine requirements for induction of systemic and mucosal CTL after nasal immunization. J Immunol. 2001;167(9):5386-5394.

7. Bradney CP, Sempowski GD, Liao HX, Haynes $\mathrm{BF}$, Staats HF. Cytokines as adjuvants for the induction of anti-human immunodeficiency virus peptide immunoglobulin $G(\operatorname{Ig} G)$ and IgA antibodies in serum and mucosal secretions after nasal immunization. J Virol. 2002; 76(2):517-524

8. Egan MA, et al. A comparative evaluation of nasal and parenteral vaccine adjuvants to elicit systemic and mucosal HIV-1 peptide-specific humoral immune responses in cynomolgus macaques. Vaccine. 2004;22(27-28):3774-3788.

9. Ackerman ME, Dugast AS, Alter G. Emerging concepts on the role of innate immunity in the prevention and control of HIV infection. Annu Rev Med. 2012;63:113-130.

10. Altfeld M, Fadda L, Frleta D, Bhardwaj N. DCs and NK cells: critical effectors in the immune response to HIV-1. Nat Rev Immunol. 2011;11(3):176-186.

11. Sui Y, et al. Vaccine-induced myeloid cell population dampens protective immunity to SIV. J Clin Invest. 2014;124(6):2538-2549.

12. Ray A, Chakraborty K, Ray P. Immunosuppressive
MDSCs induced by TLR signaling during infection and role in resolution of inflammation. Front Cell Infect Microbiol. 2013;3:52.

13. Dang Y, et al. Dendritic cell-activating vaccine adjuvants differ in the ability to elicit antitumor immunity due to an adjuvant-specific induction of immunosuppressive cells. Clin Cancer Res. 2012; 18(11):3122-3131.

14. Bunt SK, Clements VK, Hanson EM, Sinha P, Ostrand-Rosenberg S. Inflammation enhances myeloid-derived suppressor cell cross-talk by signaling through Toll-like receptor 4. J Leukoc Biol. 2009;85(6):996-1004

15. Rieber N, et al. Flagellin induces myeloid-derived suppressor cells: implications for Pseudomonas aeruginosa infection in cystic fibrosis lung disease. J Immunol. 2013;190(3):1276-1284.

16. Garg A, Spector SA. HIV type 1 gp120-induced expansion of myeloid derived suppressor cells is dependent on interleukin 6 and suppresses immunity. J Infect Dis. 2014;209(3):441-451.

17. Buchbinder SP, et al. Efficacy assessment of a cell-mediated immunity HIV-1 vaccine (the Step Study): a double-blind, randomised, placebocontrolled, test-of-concept trial. Lancet. 2008; 372(9653):1881-1893.

18. Hansen SG, et al. Profound early control of highly pathogenic SIV by an effector memory T-cell vaccine. Nature. 2011;473(7348):523-527.

\title{
Inhibiting HDAC for human hematopoietic stem cell expansion
}

Hal E. Broxmeyer

Department of Microbiology and Immunology, Indiana University School of Medicine, Indianapolis, Indiana, USA.

In this issue of the JCI, Chaurasia and colleagues report an impressive ex vivo expansion of HSCs from human cord blood (CB) using cytokines and altering epigenetic modifications. The application of this protocol provides information that has potential for clinical consideration. The enhanced expansion of CB HSCs is a substantial advance over recent work from the Chaurasia and Hoffman group, in which ex vivo production of human erythroid progenitor cells from CB was promoted by chromatin modification. Moreover, this study takes advantage of information from the rapidly emerging, but not yet fully elucidated, field of epigenetics.

\section{Limited HSCs in cord blood prevent broad clinical use}

The limited number of HSCs in single cord blood (CB) collections has been problematic for efficient engraftment in adult patients (1), hence the efforts of numerous groups to compensate for low HSC numbers by attempting to transplant double CB units, expand HSCs from CB ex vivo,

Conflict of interest: Hal E. Broxmeyer is on the Medical Scientific Advisory Board of Corduse, a cord blood banking company, and in the past has consulted for Fate Therapeutics and has received income from these companies.

Citation for this article: J Clin Invest. 2014; 124(6):2365-2368. doi:10.1172/JCI75803. increase the homing capabilities of HSCs through priming and other maneuvers, or modulate the recipient's microenvironment for more efficient engraftment (1). A number of efforts have been put forth to expand human HSCs ex vivo (2), with limited success.

\section{HDAC inhibition dramatically improves CB HSC expansion}

Epigenetics is a high-profile area of investigation (3). In this issue, Chaurasia et al. (4) followed up on their previous studies (5) by focusing on means to more effectively expand HSC populations isolated from $\mathrm{CB}$ using histone deacetylase inhib- itors (HDACIs), primarily valproic acid (VPA), either in the context of cytokine-primed CB cells or with greater effect in addition to cytokines for the 7-day period of ex vivo cell culture (Figure 1). The cytokine cocktail included stem cell factor, FLT3 ligand, thrombopoietin, and IL-3 which, together in the presence of VPA, produced a phenomenal expansion of engraftable HSCs as assayed by stateof-the-art procedures. Chaurasia and colleagues evaluated and quantitated human HSC engraftment and repopulation in sublethally irradiated NOD/SCID IL-2 receptor $\gamma \mathrm{c}$-null (NSG) mice (4). Using limiting cell dilution analysis, Chaurasia et al. determined that the frequency of SCID-repopulating cells (SRCs) after ex vivo culture of CB cells with cytokines and VPA was 1 SRC in every 31 cells, while CB cells cultured with only cytokines produced 1 SRC in 9,225 cells, and input (unexpanded CB cells) had 1 SRC in 1,115 cells. This translated to respective numbers of 32,258 SRCs from ex vivo-cultured $\mathrm{CB}$ cells in the presence of cytokines plus 

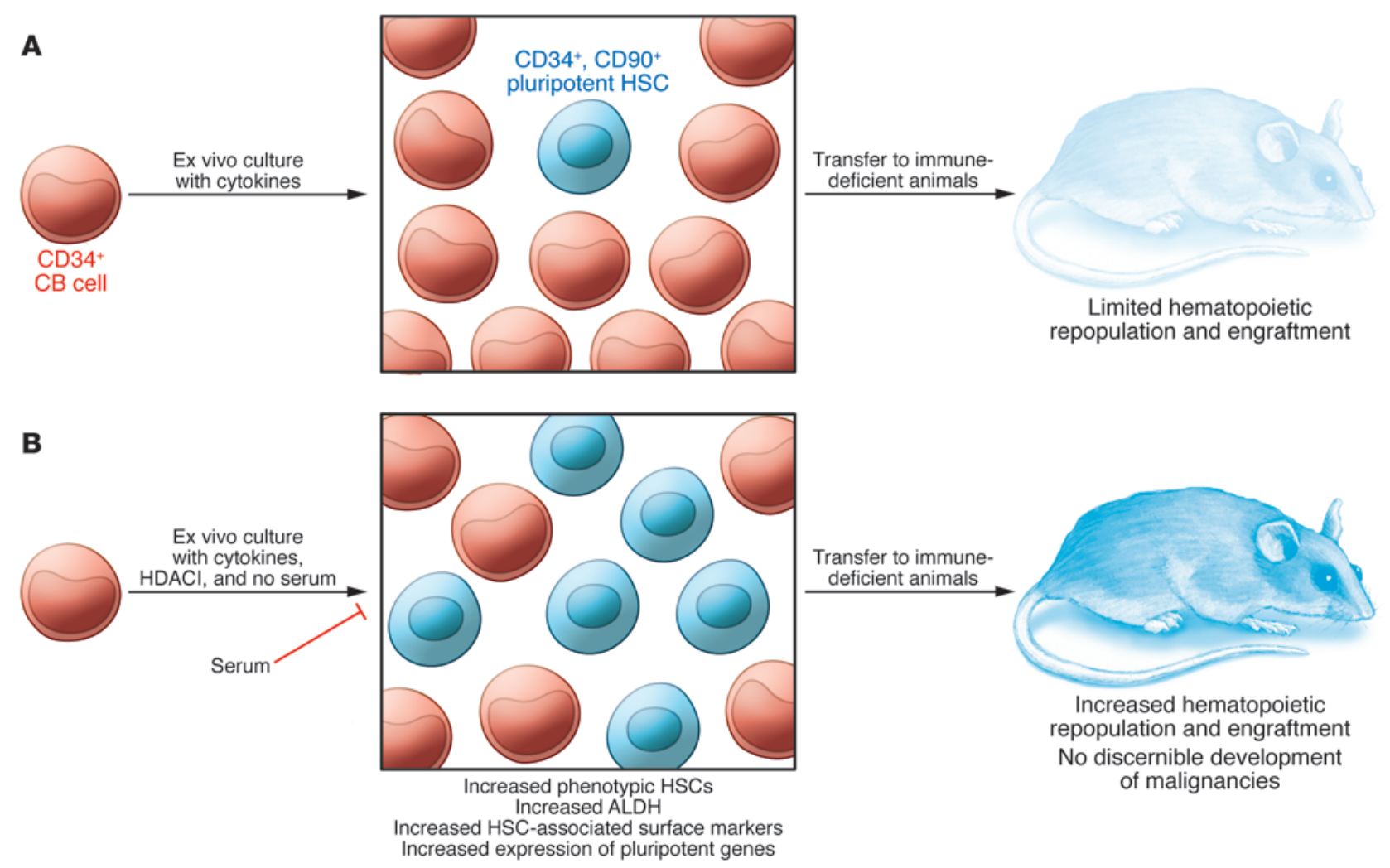

Figure 1

HDAC inhibition improves ex vivo expansion of HSCs from CB. Human CB was collected and exposed for 7 days to a cytokine cocktail or a cytokine cocktail plus $\mathrm{HDACl}$ in the presence $(\mathbf{A})$ or absence $(\mathbf{B})$ of serum. $\mathrm{CB}$ cells cultured in the presence of cytokines and $\mathrm{HDACl}$ exhibited increased numbers of cells expressing HSC phenotypes, including increased production of ALDH, upregulation of HSC surface markers CD90, C-Kit, integrin $\alpha 6$, and CXCR4, and expression of the pluripotent genes OCT4, SOX2, and NANOG (B). Surprisingly, the presence of serum decreased the efficiency of HDACl-associated HSC expansion (A). Cells expanded in in the presence of cytokines and HDACI had a much greater capacity to repopulate hematopoietic cell populations and engraft following transplantation into severely immune-deficient animals (B). The study by Chaurasia et al. (4) raises several questions for future consideration: What serum factors block full HSC expansion? What other intracellular factors are involved in epigenetic reprogramming of HSCs? Will other chromatin-remodeling agents and/or small-molecule inducers of pluripotency promote ex vivo expansion of human HSCs alone or in combination with HDACls? Can expanded HSCs with increased pluripotent gene expression be used for more efficient generation of fully programmed iPSCs?

VPA and 108 SRCs from ex vivo CB cells cultured with cytokines alone, while the input CB contained 897 SRCs. VPA-pluscytokine-expanded cells also had extensive capacity for secondary mouse repopulation, demonstrating their self-renewal activity. Thus, expansion of CB cells in the presence of cytokines alone under the conditions used in the study by Chaurasia and colleagues resulted in a loss of SRCs, while the addition of VPA with the same cytokines resulted in an approximately 36-fold increase in SRCs compared with the number of input SRCs.

The use of VPA plus cytokines also resulted in increased aldehyde dehydrogenase (ALDH) activity as well as expression of CD90, CD117 (c-Kit), CD49f (integrin $\alpha 6$ ), and CD184 (CXCR4) on the cell surface. The chemokine receptor CXCR4 binds stromal-derived factor 1 (SDF1/ CXCL12), which is known to be involved in homing of HSCs $(6,7)$. Increased expression of CXCR4 on the cytokine-plus-VPA-cultured cells was associated with increased sensitivity of these cells to chemotaxis in vitro in response to SDF1/ CXCL12 and increased HSC homing in vivo. One of the disadvantages of using CB for hematopoietic cell transplantation (HCT) is that compared with transferred bone marrow, the time to neutrophil, platelet, and immune cell reconstitution is slower (1), a phenomenon that is also apparent when two CB units are used for HCT; therefore, increased expression of CXCR4 and possibly CD49f on HSCs may allow for decreased time to engraftment. Even if enhanced CXCR4 does not improve engraftment, there are other means to decrease the engraftment period, including the use of an inhibitor of dipeptidylpeptidase 4 (DPP4). DPP4 selectively truncates a number of proteins by removing the last two amino acids from the $\mathrm{N}$ terminus, usually when the penultimate amino acid is an alanine or proline. SDF1/ CXCL12 and other cytokines have a DPP4 site, and DPP4-truncated SDF1/CXCL12 is less efficient in chemotaxis and blunts the activity of full-length SDF1/CXCL12 $(8,9)$. Moreover, inhibition of DPP4 activity with diprotin A or sitagliptin enhances the homing and engrafting capability of HSCs in mouse models $(8,10)$.

Of practical interest is the observation that ex vivo expansion of CB cells was much more efficient under serum-free conditions compared with that observed in serum-containing cultures. This dis- 
crepancy raises the question as to what exactly in the serum was acting to decrease activity for the output of HSCs. Many different cell populations, cell-derived cytokines, and other factors can act as negative regulators of $\operatorname{HSCs}(6,7,11)$. Furthermore, these molecules can act at low concentrations, especially in combination with other negative regulators. Identification of the factors involved in the downmodulating effects of serum would be informative. If the modulating factors are proteins, it will be especially important to determine whether they are full-length or DPP4-truncated forms.

\section{HDACl-dependent induction of pluripotent genes}

An interesting aspect of the study by Chaurasia and colleagues (4) is the observation that VPA induced an endogenous increase in the expression of pluripotent genes, including SOX2, OCT4, NANOG, and Z1C3. Furthermore, siRNA-mediated knockdown of OCT4, SOX2, and NANOG in $\mathrm{CB}$ cells limited the ex vivo expansion of phenotypic HSCs, verifying the role of these genes in pluripotency. Induction of these pluripotent genes in CB HSCs did not result in any obvious malignancy or promote the formation of teratomas in primary or secondary NSG mice engrafted with the expanded cells. Moreover, injection of pluripotent CB HSCs into sites that have been shown to form teratomas following embryonic stem cell (ESC) injection did not induce teratoma formation. Pluripotent genes are involved in maintaining stem cell states for ESC lines and in reprogramming somatic cells to exhibit an ESC-like state that is inherent in induced pluripotent stem cells (iPSCs), and expression of these genes is usually associated with the ability of ESCs and iPSCs to form teratomas. Of course, the possibility exists that malignancies may manifest, if and when cytokine-plus-VPA-expanded CB HSCs are used for clinical HCT, and should be kept in mind, as it might take a long time for malignant transformation to occur in patients. In the context of patient HCT, there would be a much longer time for malignancies to manifest compared with the year or so that NSG mice are monitored following human cell engraftment. Regardless of whether ex vivo cytokine-plus-VPAexpanded cells are used clinically, we are already the beneficiaries of increased knowledge about the regulation of HSC function. VPA-plus-cytokine treatment likely affects cell survival, self-renewal, and proliferation of HSCs, as OCT4 promotes survival and pluripotency in murine ESCs (12). Chaurasia et al. noted a physical association between OCT4 and NANOG in cells cultured with VPA plus cytokines (4), adding further data to support the contribution of this network of interacting transcription factors in pluripotency maintenance. Collectively, these data suggest that other genes are involved in the VPA-pluscytokine-induced effects that promote ex vivo generation and expansion of human HSCs. The more information we gather toward understanding the functional characteristics of these ex vivo-expanded cells, the more comfortable we may be in considering their clinical application for human HCT. Further informative work will be required to determine the metabolic profile of expanded CB HSCs and how they compare with unmanipulated primary HSCs, ESCs, iPSCs, and other pluripotent cells. Moreover, future studies should investigate the roles and activities of mitochondria in these different pluripotent cell types. Based on the role of HDACIs in promoting HSC expansion, a possible group of proteins to investigate includes members of the sirtuin family of deacetylases (13), including SIRT1, which has been linked to NANOG expression, p53 subcellular localization, and mitochondrial function in murine ESCs (14-16), as well as to hematopoietic cell differentiation during embryogenesis and in adult mice (17). These SIRT1-associated effects are especially apparent under stressful conditions, which ex vivo culture of HSCs can certainly be considered. Other potential players that could be involved in chromatin remodeling of HSCs include DEK, a unique protein that is involved in HSC regulation and hematopoietic progenitor cell biology (18).

Of the eight HDACIs tested in the ex vivo system used by Chaurasia and colleagues, three (VPA, scriptaid, and CAY10433) enhanced cytokine-stimulated HSC expansion (4). Understanding why some HDACIs worked and why others were less effective or failed may shed more light on the mechanisms underlying the reprogramming of CB HSCs. Various chemical approaches are being applied to stem cell biology (19), and some of these approaches, alone or in combination with VPA or other HDACIs, may be of value in deciphering how to increase the numbers and/or potency of human HSCs for therapeutic use. Reprogramming of somatic cells, such as $\mathrm{CB}$ CD $34^{+}$cells, to an iPSC state results in colonies that are morphologically indistinguishable from ESCs; however, some iPSC colonies contain only partially reprogrammed cells (20). In this context, it would be reasonable to determine whether the CB HSCs produced ex vivo in the presence of VPA and cytokines, which already have enhanced expression of OCT4, SOX2, and NANOG, can be more efficiently induced toward fully reprogrammed iPSCs. Information on how to maximize the generation of iPSCs is of great scientific as well as potential practical interest in the context of regenerative medicine.

\section{Conclusions}

The study by Chaurasia et al. (4) presents important steps toward further understanding HSC biology and how to potentially manipulate these cells for therapeutic advantage. There have now been over 30,000 CB HCTs performed (1), and the means to enhance the efficacy of this procedure could benefit many patients with malignant and nonmalignant disorders who cannot otherwise find another appropriate source of HLA-matched allogeneic HSCs for HCT.

\section{Acknowledgments}

Studies cited in the reference section by the author's laboratory were supported by Public Health Service Grants from the NIH (R01 HL056416, R01 HL67384, R01 HL112669, and P01 DK090948).

Address correspondence to: Hal E. Broxmeyer, Indiana University School of Medicine, Department of Microbiology and Immunology, 950 West Walnut Street, R2-302, Indianapolis, Indiana 462025181, USA. Phone: 317.274.7510; Fax: 317.274.7592; E-mail: hbroxmey@iupui.edu.

1. Ballen KK, Gluckman E, Broxmeyer HE. Umbilical cord blood transplantation: the first 25 years and beyond. Blood. 2013;122(4):491-498.

2. Flores-Guzman P, Fernandez-Sanchez V, Mayani H. Concise review: ex vivo expansion of cord bloodderived hematopoietic stem and progenitor cells: basic principles, experimental approaches, and impact in regenerative medicine. Stem Cells Transl Med. 2013;2(11):830-838.

3. Felsenfeld G. A brief history of epigenetics. Cold Spring Harb Perspect Biol. 2014;6(1):a018200.

4. Chaurasia P, Gajzer DC, Schaniel C, D'Souza S, Hoffman R. Epigenetic reprogramming induces the expansion of cord blood stem cells. J Clin Invest. 2014;124(6):2378-2395.

5. Chaurasia P, Berenzon D, Hoffman R. Chromatinmodifying agents promote the ex vivo production of functional human erythroid progenitor cells. 
Blood. 2011;117(17):4632-4641.

6. Shaheen M, Broxmeyer HE. Hematopoietic cytokines and growth factors. In: Broxmeyer HE, eds. Cord Blood Biology, Transplantation, Banking, and Regulation. Bethesda, Maryland, USA:AABB Press; 2011:35-74

7. Shaheen M, Broxmeyer HE. Principles of cytokine signaling. In: Hoffman R, Benz EJ Jr, Silberstein LE, Heslop H, Weitz JI, Anastasi J, eds. Hema tology: Basic Principles and Practice. 6th ed. Philadelphia, Pennsylania, USA: Elsevier Saunders; 2013:136-146.

8. Broxmeyer HE, et al. Dipeptidylpeptidase 4 negatively regulates colony-stimulating factor activity and stress hematopoiesis. Nat Med. 2012; 18(12):1786-1796

9. Ou X, O'Leary HA, Broxmeyer HE. Implications of DPP4 modification of proteins that regulate stem/progenitor and more mature cell types. Blood. 2013;122(2):161-169.

10. Christopherson KW 2nd, Hangoc G, Mantel CR,
Broxmeyer HE. Modulation of hematopoietic stem cell homing and engraftment by CD26. Science. 2004;305(5686):1000-1003.

11. Csaszar E, et al. Rapid expansion of human hematopoietic stem cells by automated control of inhibitory feedback signaling. Cell Stem Cell. 2012; 10(2):218-229

12. Guo Y, Mantel C, Hromas RA, Broxmeyer HE. Oct 4 is critical for survival/antiapoptosis of murine embryonic stem cells subjected to stress, effects associated with Stat3/Survivin. Stem Cells. 2008; 26(1):30-34.

13. Sack MN, Finkel T. Mitochondrial metabolism, sirtuins, and aging. Cold Spring Harb Perspect Biol. 2012;4(12):a013102.

14. Han M-K, Song EK, Guo Y, Ou X, Mantel C, Broxmeyer HE. SIRT1 regulates apoptosis and Nanog expression in mouse embryonic stem cells by controlling p53 subcellular localization. Cell Stem Cell. 2008;2(3):241-251

15. Zhang ZN, Chung SK, Xu Z, Xu Y. Oct4 maintains the pluripotency of human embryonic stem cells by inactivating p53 through Sirt1-mediated deacetylation. Stem Cells. 2014;32(1):157-165.

16. Ou X, Lee MR, Huang X, Messina-Graham S, Broxmeyer HE. SIRT1 positively regulates autophagy and mitochondria function in embryonic stem cells under oxidative stress. Stem Cells. 2014; 32(5):1183-1194.

17. Ou X, et al. SIRT1 deficiency compromises mouse embryonic stem cell hematopoietic differentiation, and embryonic and adult hematopoiesis in the mouse. Blood. 2011;117(2):440-450.

18. Broxmeyer HE, et al. A role for DEK in stem/progenitor cell biology. Stem Cells. 2013;31(8):1447-1453.

19. Li W, Li K, Wei W, Ding S. Chemical approaches to stem cell biology and therapeutics. Cell Stem Cell. 2013;13(3):270-283

20. Lee MR, et al. Epigenetic regulation of Nanog by miR-302 cluster-MBD2 completes induced pluripotent stem cell reprogramming. Stem Cells. 2013;31(4):666-681.

\title{
Unraveling the relationship between microbial translocation and systemic immune activation in HIV infection
}

\author{
Liang Shan ${ }^{1}$ and Robert F. Siliciano ${ }^{2,3}$
}

${ }^{1}$ Department of Immunobiology, Yale University, New Haven, Connecticut, USA. ${ }^{2}$ Department of Medicine, Johns Hopkins University School of Medicine, Baltimore, Maryland, USA. ${ }^{3}$ Howard Hughes Medical Institute, Baltimore, Maryland, USA.

Chronic immune activation is a key factor in HIV-1 disease progression. The translocation of microbial products from the intestinal lumen into the systemic circulation occurs during HIV-1 infection and is associated closely with immune activation; however, it has not been determined conclusively whether microbial translocation drives immune activation or occurs as a consequence of HIV-1 infection. In an important study in this issue of the $J C I$, Kristoff and colleagues describe the role of microbial translocation in producing immune activation in an animal model of HIV-1 infection, SIV infection of pigtailed macaques. Blocking translocation of intestinal bacterial LPS into the circulation dramatically reduced $T$ cell activation and proliferation, production of proinflammatory cytokines, and plasma SIV RNA levels. This study directly demonstrates that microbial translocation promotes the systemic immune activation associated with HIV-1/SIV infection.

\section{Immune activation and disease progression in HIV-1 infection}

In humans, the gut-associated lymphoid tissue (GALT) contains a large number of activated $\mathrm{CD}^{+}{ }^{+} \mathrm{CR} 5^{+} \mathrm{T}$ cells, thus serving as a major site of viral replication and $\mathrm{CD}^{+} \mathrm{T}$ cell depletion early in the course of HIV-1 infection $(1,2)$. In contrast to the rapid depletion of intestinal $\mathrm{CD} 4^{+} \mathrm{T}$ cells, the decline of $\mathrm{CD}^{+} \mathrm{T}$ cells in peripheral

Conflict of interest: The authors have declared that no conflict of interest exists.

Citation for this article: $J$ Clin Invest. 2014;

124(6):2368-2371. doi:10.1172/JCI75799. blood is much slower and less extensive during acute HIV-1 infection, suggesting that the majority of peripheral $\mathrm{CD} 4^{+} \mathrm{T}$ cells are nonpermissive at this stage $(2,3)$. During the chronic phase of infection, systemic immune activation is very strongly correlated with disease progression (4). Activation of $\mathrm{CD}^{+} \mathrm{T}$ cells increases coreceptor expression and renders these cells more susceptible to HIV-1 infection, and these cycles of activation and infection may drive progression of disease to AIDS (5). In contrast, SIV infection in natural host species very rarely progresses to AIDS and does not result in increased levels of immune activation, despite high levels of virus replication $(6,7)$. Infection and depletion of $\mathrm{CD}^{+} \mathrm{T}$ cells in GALT can be observed in both SIV and HIV-1 infection; however, natural host species infected with SIV do not often develop systemic immune activation, despite loss of GALT CD4 ${ }^{+} \mathrm{T}$ cells $(6,7)$, suggesting that factors other than direct infection and mucosal $\mathrm{CD} 4^{+} \mathrm{T}$ cell loss are essential for the increased degree of immune activation seen in HIV-1 infection.

\section{Microbial translocation in HIV-1 infection}

Translocation of microbial products from the lumen of the gastrointestinal tract into the circulation often occurs in HIV-1infected individuals and is closely associated with systemic immune activation. Many studies have shown that HIV-1infected individuals have elevated plasma levels of LPS and soluble CD14, which is indicative of LPS-induced monocyte and macrophage activation (8). Plasma levels of other microbial products, such as bacterial DNA and flagellin, are also increased in HIV-1-infected individuals compared with healthy controls $(8,9)$. Furthermore, there is a negative correlation between plasma 